\title{
Successful treatment of atopic dermatitis with house dust mite sublingual immunotherapy tablets
}

\author{
Ploykarn Kiatiwat, ${ }^{1}$ Wat Mitthamsiri, ${ }^{1}$ Tadech Boonpiyathad, ${ }^{1}$ Panitan Pradubpongsa, ${ }^{1}$ Atik Sangasapaviliya ${ }^{1}$
}

\begin{abstract}
Background: Atopic dermatitis (AD) is the most common chronic inflammatory skin disease. Allergen-specific immunotherapy is a treatment option for selected patients with severe AD sensitization to house dust mites (HDM).
\end{abstract}

Objective: To report the first case of successful treatment with HDM sublingual immunotherapy (SLIT) tablets in patients with severe $\mathrm{AD}$.

Method: A Thai male patient with HDM sensitization and severe AD who had not responded to topical corticosteroids and calcineurin inhibitors underwent 1 month of HDM subcutaneous immunotherapy (SCIT), after which his skin symptoms were minimally improved. He lost follow-up SCIT and the symptoms worsened, with large wheal lesions appearing at the SCIT injection site, so we decided to switch from SCIT to HDM SLIT tablets.

Results: After the SLIT treatment, the AD and skin lesions improved and the medication could be stopped.

Conclusion: HDM SLIT might be an alternative treatment in patients with HDM sensitization and severe AD who are refractory to conventional treatment.

Key words: house dust mites; allergen-specific immunotherapy; atopic dermatitis; SLIT; SCORAD

From:

${ }^{1}$ Division of Allergy and Clinical Immunology,

Department of Medicine, Phramongkutklao Hospital,

Bangkok, Thailand
Corresponding author:

Atik Sangasapaviliya

Division of Allergy and Clinical Immunology,

Department of Medicine, Phramongkutklao Hospital

and Phramongkutklao College of Medicine, Bangkok, Thailand

E-mail:pmk.atik@gmail.com

\section{Introduction}

Atopic dermatitis $(\mathrm{AD})$ is one of the most common chronic or relapsing inflammatory skin diseases, with significant morbidity and highly intractable pruritus, and is often associated with other atopic disorders, especially asthma and allergic rhinitis. ${ }^{1} \mathrm{AD}$ commonly affects children and to a lesser extent adults. The prevalence of $\mathrm{AD}$ worldwide is about 10-20\% among children and 1-3\% among adults. ${ }^{2}$ Prevalence is steadily increasing by exposure to pollutants and allergens, especially house dust mites (HDM). In all, $80-85 \%$ of patients with $\mathrm{AD}$ have increased aeroallergen-specific immunoglobulin $\mathrm{E}(\mathrm{IgE})$ antibodies. ${ }^{3}$

AD treatment includes skin hydration, topical agents (steroids and topical calcineurin inhibitors), phototherapy, systemic immunomodulating agents (systemic steroids, cyclosporin and azathioprine) and monoclonal antibody (dupilumab). ${ }^{4}$ Systemic immunomodulating agents are generally reserved for moderate to severe $\mathrm{AD}$ because of their systemic toxicities, such as adrenal suppression, diabetes, renal toxicity, liver toxicity and myelosuppression. ${ }^{4}$ The Joint Task Force on Practice Parameters states that allergen-specific immunotherapy (AIT) might be considered among selected patients with $\mathrm{AD}$ and aeroallergen sensitivity. In contrast, the American Academy of Dermatology guidelines discuss the literature for both sublingual and injection immunotherapy, concluding that the data do not support the recommendation for AIT use among patients with $\mathrm{AD}$ at this time. ${ }^{5}$

AIT can induce the reinstatement of tolerance toward allergens and represents a disease-modifying treatment. ${ }^{6}$ One of the most important mechanisms in AIT is the induction of regulatory $\mathrm{T}$ and $\mathrm{B}$ cells (Tregs and Bregs). AIT upregulates activated allergen-specific Tregs and downregulates dysfunctional allergen-specific Tregs. ${ }^{7}$ Tregs release interleukin 10, 
block mast cell degranulation and engage cytotoxic T lymphocyte antigen 4 and programmed cell death protein $1 .{ }^{8}$ Bregs can suppress effector $\mathrm{T}$ cells, inhibit dendritic cell maturation and induce Tregs and IgG4 antibodies. ${ }^{9}$ Moreover, type 2 innate lymphoid cells are significantly reduced following AIT. ${ }^{10}$ Mast cells and basophils decrease responsiveness to the allergen in the desensitization stage, measured by decreased basophil activation. ${ }^{11}$

AIT may be a treatment option for selected patients with $\mathrm{AD}$ who have proven sensitization to HDM and severe eczema that cannot be controlled with conventional therapies. Here, we report the first case of successful treatment with HDM sublingual immunotherapy (SLIT) tablets in a patient with severe AD. HDM SLIT tablets improved the scoring atopic dermatitis (SCORAD) index and skin lesions, slightly decreased serum total IgE levels and reduced medication use. The study protocol was approved by the Institutional Review Board of the Royal Thai Army's Ethics Committee and the patient gave written informed consent to participate.

\section{Case report}

A 17-year-old Thai male with a history of $\mathrm{AD}$ and allergic rhinitis from 10 years old visited and had follow-up treatment at the Pediatric Allergy Clinic, Phramongkutklao Hospital, Bangkok, Thailand. In the last 3 years, he had developed progressive intractable pruritic eczema on the face, neck, back, abdomen and all extremities, predominantly on both flexural areas. On treating the AD with topical corticosteroids such as betamethasone cream and an oral antihistamine, his symptoms did not improve. The first time (Visit 0) at the Medicine Allergy Clinic, his SCORAD was 75 (Figure 1A). The patient had been treated with emollients $(0.1 \%$ triamcinolone lotion applied to the body, $0.02 \%$ triamcinolone cream applied to the face, $0.1 \%$ tacrolimus ointment applied to the face and body) and oral antihistamine and prednisolone $20 \mathrm{mg}$ /day for 3 days. At baseline, his serum total IgE level was $6120 \mathrm{IU} / \mathrm{ml}$ (normal range 150-1000 IU/ml) and serum specific IgE levels to various allergens were: mixed HDM, $94 \mathrm{kU} / \mathrm{l}$; acacia, $5.5 \mathrm{kU} / \mathrm{l}$; Candida albicans, $13.4 \mathrm{kU} / \mathrm{l}$; mixed grass, $3.1 \mathrm{kU} / \mathrm{l}$;

\section{(A) Visit 0 (at baseline)}

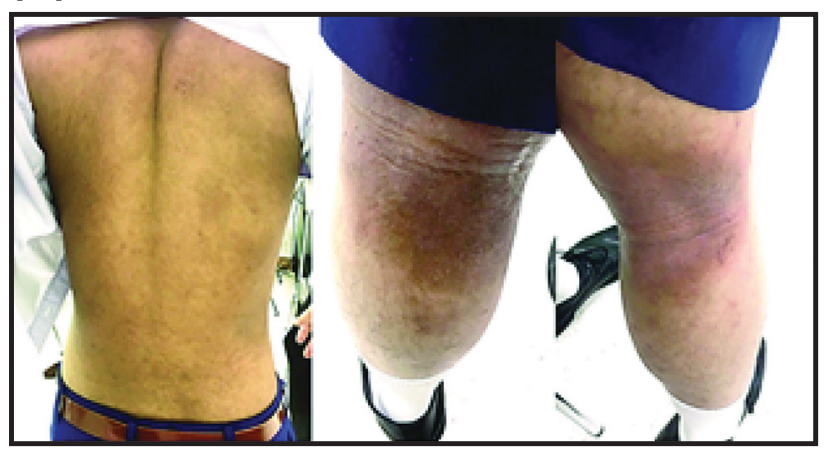

(B) Visit 2 (2 months after baseline)

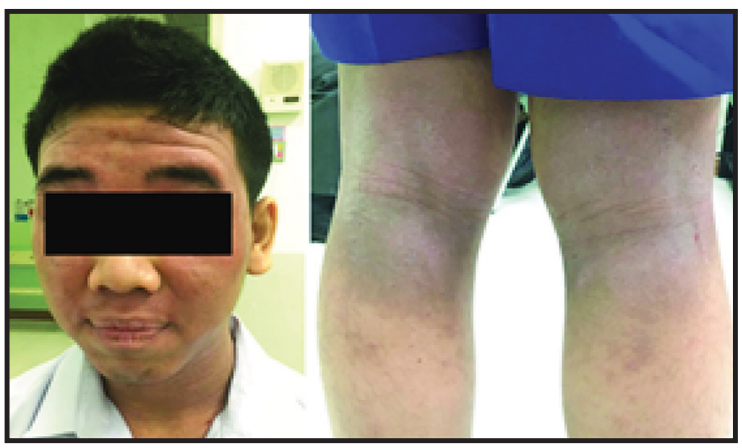

(C) Visit 3 (4 months after baseline and started SLIT)

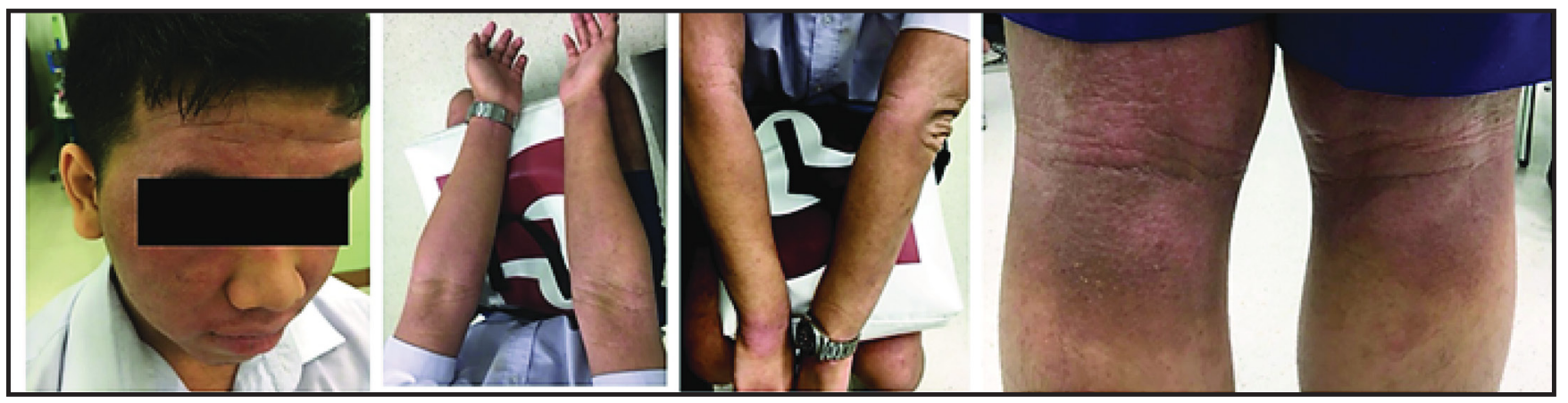

Figure 1. Photos of a 17-year-old Thai male with severe atopic dermatitis showing skin lesion improvement during treatment with sublingual immunotherapy (SLIT) tablets.

(A) Visit 0: patient presented with progressive intractable purification eczema on face, neck, back, abdomen and extremities, predominantly on both flexural areas, and received topical steroids, topical calcineurin inhibitors and short-course oral steroids; his scoring atopic dermatitis (SCORAD) index was 75 at baseline.

(B) Visit 2 (2 months from baseline and 1 month after subcutaneous immunotherapy): the SCORAD was 55.6; decreased erythematous plaques with scale on wrists, thigh, neck and back but no lesion changes on the face.

(C) Visit 3 (4 months from baseline and switched to SLIT): the SCORAD was 65.8; widespread erythematous, lichenified plaques, oozing blood and encrusted entire both arms, thighs, abdominal wall; progressive lesions on face. 
(D) Visit 4 (1 month after SLIT)

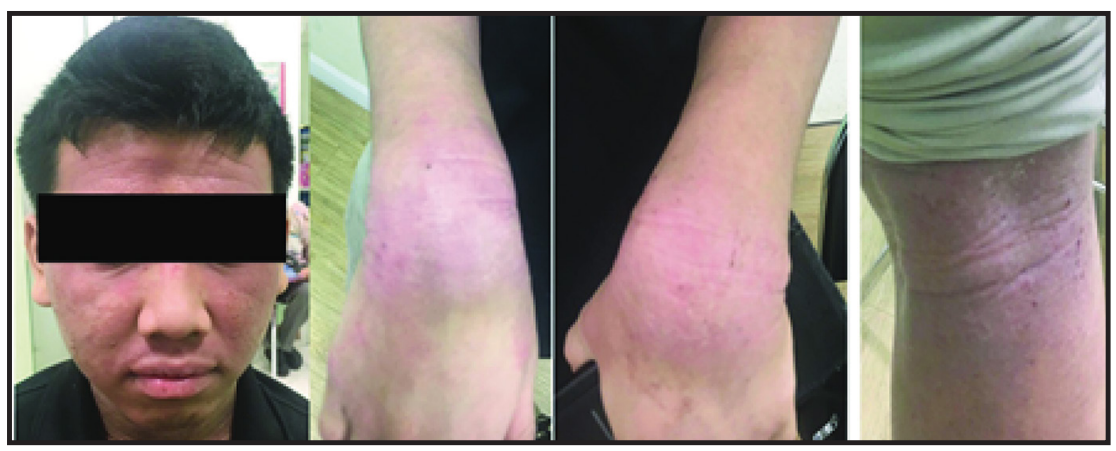

(F) Visit 6 (7 months after SLIT)

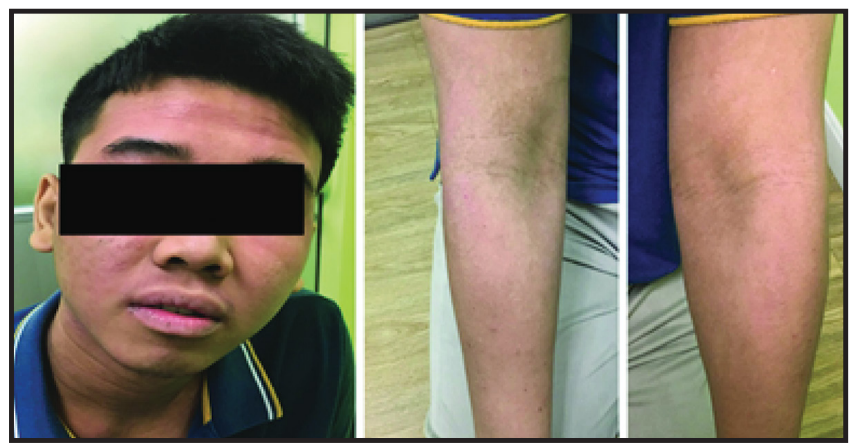

(E) Visit 5 (4 months after SLIT)

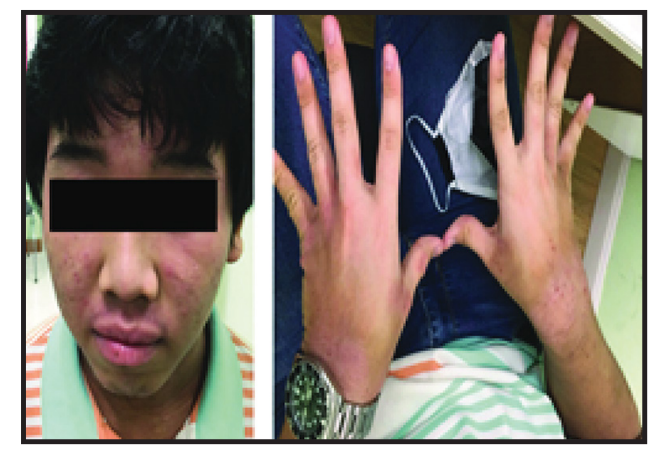

Figure 1. (Continued)

(D) Visit 4 (5 months from baseline and 1 month after SLIT): the SCORAD was 57.2; resolution of lesions on the abdominal wall and both arms; decrease in the area of erythematous plaques and encrusting on the face, neck and both thighs; a new active lesion on his right wrist.

(E) Visit 5 (8 months from baseline and 4 months after SLIT): the SCORAD was 36.7; improved lesions (some hyperpigmented plaque and encrusted) on face, neck and wrist.

(F) Visit 6 (11 months from baseline and 7 months after SLIT): the SCORAD was 12.8 and there were no active skin lesions.

German cockroach, $3.1 \mathrm{kU} / \mathrm{l}$; mixed tree, $1 \mathrm{kU} / \mathrm{l}$; other aeroallergens, < $0.1 \mathrm{kU} / \mathrm{l}$. After 1 month of treatment (Visit 1 ), his skin symptoms had worsened and the SCORAD was 63 (Figure 2). We talked to the patient and decided to start mixed HDM subcutaneous immunotherapy (SCIT) with $0.25 \mathrm{ml}$ of Dermatophagoides pteronyssinus (1:1000, $5 \mathrm{AU} / \mathrm{ml})$ plus 0.25 $\mathrm{ml}$ of $D$. farina (Der f, 1:1000, $5 \mathrm{AU} / \mathrm{ml}$ ) (ALK, Abello, USA). One month after weekly conventional SCIT (Visit 2), his SCORAD had decreased to 55.6 (Figure 1B).

Two months later (Visit 3), his symptoms had worsened because he missed the follow-up SCIT injection after attending a Reserve Officer Training Course. He also misused the topical steroids and emollients. Furthermore, he was in contact with high levels of pollution and dust at his new workplace. His SCORAD increased to 65.8 and there was widespread $\mathrm{AD}$ that included erythematous, lichenified plaques, oozing blood and encrusted entire upper arms and abdominal wall (Figure 1C). In addition, the entire injected site presented large wheal lesions. We decided to stop SCIT and changed to HDM SLIT (Acarizax ${ }^{\circledast} 12$ SQ; $15 \mu \mathrm{g}$ of combined Der f1 + Der p1 and $15 \mu \mathrm{g}$ of Der $\mathrm{f} 2$ + Der p2 per tablet; Abbot, Denmark), 1 tablet once daily. The other treatments, such as topical corticosteroid, topical tacrolimus, oral antihistamine and emollients, were continued. One month after treatment with SLIT (Visit 4), his skin symptoms minimally improved

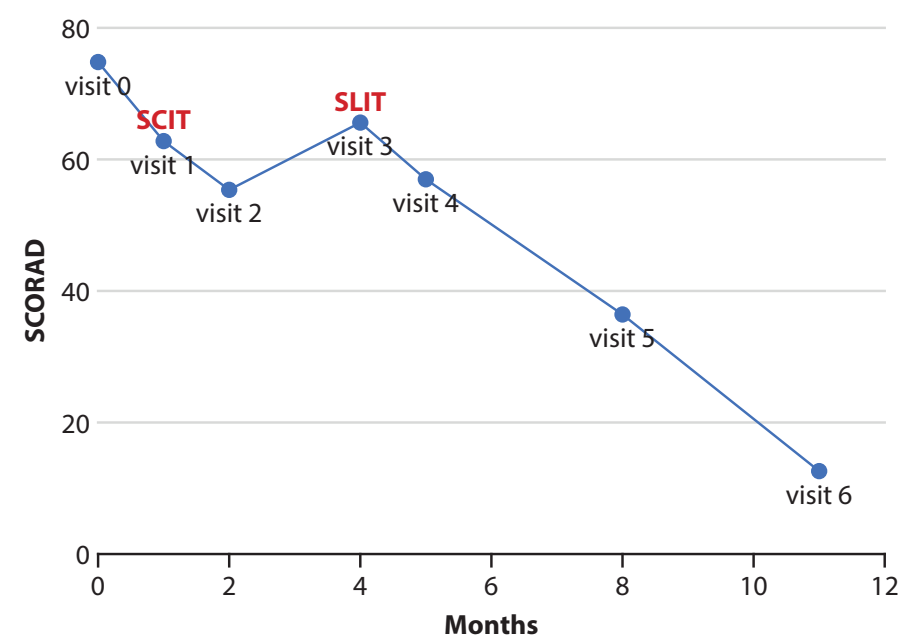

Figure 2. Changes in the scoring atopic dermatitis (SCORAD) index during treatment. SCIT, subcutaneous immunotherapy; SLIT, sublingual immunotherapy.

and his SCORAD was 57.2 (Figure 1D). Four months after treatment with SLIT (Visit 5), his skin symptoms were better and the SCORAD had decreased to 36.7 (Figure 1E). Seven months after treatment with SLIT (Visit 6), his SCORAD had decreased to 12.8 (Figure 1F). He used only SLIT tablets 
to control the $\mathrm{AD}$ and stopped using topical corticosteroid and topical tacrolimus. His serum total IgE level had decreased to $5,055 \mathrm{IU} / \mathrm{ml}$ and the serum specific IgE to mixed HDM had decreased to $70.87 \mathrm{kU} / 1$.

\section{Discussion}

$\mathrm{AD}$ is a multifactorial disease. The pathogenesis of $\mathrm{AD}$ is primarily driven by the Th2 pathway associated with IgE-mediated sensitization but with significant contributions from the Th22 pathway, the Th1 and Th17 axes and JAK/STAT signaling. ${ }^{12}$ Genetic, immunological, skin barrier dysfunction and environmental factors were observed to be the greatest risk factors for eczema development. ${ }^{12} \mathrm{HDM}$, one of the most common allergens that can penetrate damaged skin barriers, promote the polarization of a type 2 immune response, elevate the sensitization of allergen-specific $\operatorname{IgE}$ and aggravate the symptoms of $\mathrm{AD} .{ }^{13} \mathrm{HDM}$ can induce $\mathrm{AD}$ from both immediate and delayed allergic reactions. Severe $\mathrm{AD}$ correlates with the degree of sensitization to aeroallergens. ${ }^{13}$

AIT is the only treatment to modify the natural progression of the allergic disease. However, the role of AIT in AD is controversial. ${ }^{14}$ Recent studies show that AIT can improve the clinical outcome of AD. A randomized placebo-controlled trial (RPCT) of 24 stable AD adults reported that HDM SCIT significantly reduced $\mathrm{AD}$ symptoms after 4 months. ${ }^{15}$ The results of an RPCT from China showed that D. farina SLIT drops could decrease the symptom and total medication scores in moderate AD patients after 36 weeks of treatment. ${ }^{16}$ A study of 34 adult Korean AD patients reported that SLIT (D. pteronyssinus + D. farina extract drops) showed efficacy and tolerability. ${ }^{17}$ An RPCT in 239 AD children treated with SLIT (D. farina drops) showed the same result. ${ }^{18}$ The onset and dose-related efficacy of HDM SLIT tablets was 8 weeks after treatment compared to placebo. ${ }^{19}$ Another study reported that the beginning of the SLIT outcome was usually found 4-8 weeks after initiation. ${ }^{20}$ However, many studies have demonstrated that AIT was not beneficial in $\mathrm{AD}$ management. Thus, the use of AIT in AD is considered to be investigational and suggested only as an add-on therapy in selected patients who are non-responsive to traditional therapy. ${ }^{21-23}$

In this report, our $\mathrm{AD}$ patient with multiple aeroallergen sensitization had a beneficial impact from HDM SLIT. The reason might be that the patient had severely uncontrolled $\mathrm{AD}$ and the disease was aggravated by HDM exposure. Furthermore, HDM SLIT tablets were reported to be a successful treatment for HDM allergic patients. Moreover, the patient had good compliance and no side effects from the HDM SLIT tablets. We report the first case of successful treatment with HDM SLIT tablets in a patient with severe AD. The HDM SLIT tablets improved the SCORAD and skin lesions and slightly decreased total IgE levels and medication use in the severe AD patient. The HDM SLIT tablets might be an alternative treatment in severe $\mathrm{AD}$ patients who are refractory to conventional therapy. A further, well-designed controlled trial of HDM SLIT tablets should be conducted to show the benefit of the treatment in patients with severe AD.

\section{References}

1. Saini S, Pansare M. New Insights and Treatments in Atopic Dermatitis. Pediatr Clin North Am. 2019;66:1021-33.

2. Kowalska-Oledzka E, Czarnecka M, Baran A. Epidemiology of atopic dermatitis in Europe. J Drug Assess. 2019;8:126-8.

3. Mohn CH, Blix HS, Halvorsen JA, Nafstad P, Valberg M, Lagerlov P. Incidence Trends of Atopic Dermatitis in Infancy and Early Childhood in a Nationwide Prescription Registry Study in Norway. JAMA Netw Open. 2018;1:e184145.

4. Czarnowicki T, Krueger JG, Guttman-Yassky E. Novel concepts of prevention and treatment of atopic dermatitis through barrier and immune manipulations with implications for the atopic march. J Allergy Clin Immunol. 2017;139:1723-34.

5. Eichenfield LF, Ahluwalia J, Waldman A, Borok J, Udkoff J, Boguniewicz M. Current guidelines for the evaluation and management of atopic dermatitis: A comparison of the Joint Task Force Practice Parameter and American Academy of Dermatology guidelines. J Allergy Clin Immunol. 2017;139:S49-S57.

6. Globinska A, Boonpiyathad T, Satitsuksanoa P, Kleuskens M, van de Veen W, Sokolowska M, et al. Mechanisms of allergen-specific immunotherapy: Diverse mechanisms of immune tolerance to allergens. Ann Allergy Asthma Immunol. 2018;121:306-12.

7. Boonpiyathad T, Sokolowska M, Morita H, Ruckert B, Kast JI, Wawrzyniak $\mathrm{M}$, et al. Der p 1-specific regulatory T-cell response during house dust mite allergen immunotherapy. Allergy. 2019;74:976-85.

8. Boonpiyathad T, Sozener ZC, Akdis M, Akdis CA. The role of Treg cell subsets in allergic disease. Asian Pac J Allergy Immunol. 2020;38:139-49.

9. Boonpiyathad T, Satitsuksanoa P, Akdis M, Akdis CA. Il-10 producing T and B cells in allergy. Semin Immunol. 2019;44:101326.

10. Mitthamsiri W, Pradubpongsa P, Sangasapaviliya A, Boonpiyathad T. Decreased CRTH2 Expression and Response to Allergen Re-stimulation on Innate Lymphoid Cells in Patients With Allergen-Specific Immunotherapy. Allergy Asthma Immunol Res. 2018;10:662-74.

11. Sokolowska M, Boonpiyathad T, Escribese MM, Barber D. Allergen -specific immunotherapy: Power of adjuvants and novel predictive biomarkers. Allergy. 2019;74:2061-3.

12. Munera-Campos M, Carrascosa JM. Innovation in Atopic Dermatitis: From Pathogenesis to Treatment. Actas Dermosifiliogr. 2020;111:205-21.

13. Friedmann PS. The role of dust mite antigen sensitization and atopic dermatitis. Clin Exp Allergy. 1999;29:869-72.

14. Lee J, Park CO, Lee KH. Specific immunotherapy in atopic dermatitis. Allergy Asthma Immunol Res. 2015;7:221-9.

15. Leroy BP, Boden G, Lachapelle JM, Jacquemin MG, Saint-Remy JM. A novel therapy for atopic dermatitis with allergen-antibody complexes: a double-blind, placebo-controlled study. J Am Acad Dermatol. 1993;28: 232-9.

16. Sanchez Caraballo JM, Cardona Villa R. Clinical and immunological changes of immunotherapy in patients with atopic dermatitis: randomized controlled trial. ISRN Allergy. 2012;2012:183983.

17. You HS, Yang MY, Kim GW, Cho HH, Kim WJ, Mun JH, et al. Effectiveness of Specific Sublingual Immunotherapy in Korean Patients with Atopic Dermatitis. Ann Dermatol. 2017;29:1-5.

18. Liu L, Chen J, Xu J, Yang Q, Gu C, Ni C, et al. Sublingual immunotherapy of atopic dermatitis in mite-sensitized patients: a multi-centre, randomized, double-blind, placebo-controlled study. Artif Cells Nanomed Biotechnol. 2019;47:3540-7.

19. Nolte H, Maloney J, Nelson HS, Bernstein DI, Lu S, Li Z, et al. Onset and dose-related efficacy of house dust mite sublingual immunotherapy tablets in an environmental exposure chamber. J Allergy Clin Immunol. 2015;135:1494-501 e6.

20. Klimek L, Mosbech H, Zieglmayer P, Rehm D, Stage BS, Demoly P. SQ house dust mite (HDM) SLIT-tablet provides clinical improvement in HDM-induced allergic rhinitis. Expert Rev Clin Immunol. 2016;12:369-77.

21. Cox L, Calderon MA. Allergen Immunotherapy for Atopic Dermatitis: Is There Room for Debate? J Allergy Clin Immunol Pract. 2016;4:435-44.

22. Ridolo E, Martignago I, Riario-Sforza GG, Incorvaia C. Allergen immunotherapy in atopic dermatitis. Expert Rev Clin Immunol. 2018;14: $61-8$.

23. Caminiti L, Panasiti I, Landi M, De Filippo M, Olcese R, Ciprandi G, et al. Allergen immunotherapy in atopic dermatitis: Light and shadow in children. Pediatr Allergy Immunol. 2020;31 Suppl 26:46-8. 